\title{
Peak Voluntary Cough Flow and Oropharyngeal Dysphagia as Risk Factors for Pneumonia
}

\author{
Jayoon Choi, MD, Sora Baek, MD, PhD, Gowun Kim, MD, PhD, Hee-won Park, MD
}

Department of Rehabilitation Medicine, Kangwon National University Hospital, Kangwon National University School of Medicine, Chuncheon, Korea

Objective To investigate the relationship between voluntary peak cough flow (PCF), oropharyngeal dysphagia, and pneumonia in patients who were evaluated with videofluoroscopic swallowing study (VFSS).

Methods Patients who underwent both VFSS and PCF measurement on the same day were enrolled retrospectively $(n=821)$. Pneumonia $(n=138)$ and control $(n=683)$ groups were assigned based on presence of pneumonia within 1 month from the date of VFSS assessment. In addition, sex, age ( $<65$ and $\geq 65$ years), preceding conditions, modified Barthel Index (MBI), Mini-Mental State Examination (MMSE), PCF value ( $<160, \geq 160$ and $<270$, and $\geq 270 \mathrm{~L} / \mathrm{min}$ ), and presence of aspiration/penetration on VFSS were reviewed.

Results Pneumonia group was more likely to be male ( $n=108 ; 78.3 \%), \geq 65$ years $(n=121 ; 87.7 \%)$, with neurodegenerative $(\mathrm{n}=25 ; 18.1 \%)$ or other miscellaneous diseases $(\mathrm{n}=50 ; 36.2 \%)$, and in poor functional level with lower value of MBI (39.1 \pm 26.59$)$. However, MMSE was not significantly different in comparison to that of the control group. The pneumonia group was also more likely to have dysphagia (82.6\%) and lower value of PCF ( $<160 \mathrm{~L} / \mathrm{min}, 70.3 \%)$. In multivariable logistic regression analysis, male sex (odd ratio $[\mathrm{OR}]=6.62 ; 95 \%$ confidence interval [CI], 2.70-16.26), other miscellaneous diseases as preceding conditions ( $\mathrm{OR}=2.52$; 95\% CI, 1.14-5.58), dysphagia (OR=3.82; 95\% CI, 1.42-10.23), and $\mathrm{PCF}<160 \mathrm{~L} / \mathrm{min}$ (OR=14.34; 95\% CI, 1.84-111.60) were factors significantly related with pneumonia.

Conclusion Impaired swallowing and coughing function showed an independent association with the development of pneumonia. Patients with PCF $<160 \mathrm{~L} / \mathrm{min}$ require more attention with lung care and should be encouraged with voluntary coughing strategy to prevent possible pulmonary complications.

Keywords Cough, Peak expiratory flow rate, Deglutition disorders, Pneumonia

\section{INTRODUCTION}

Pneumonia is a commonly encountered disorder with high disease burden [1] and has an increased incidence in older individuals, men, those with chronic lung disease, or immobility [2]. The sequelae of pneumonia could

Received March 31, 2021; Revised June 16, 2021; Accepted August 2, 2021; Published online December 31, 2021 Corresponding author: Sora Baek

Department of Rehabilitation Medicine, Kangwon National University Hospital, Kangwon National University School of Medicine, 156 Baengnyeongro, Chuncheon 24289, Korea. Tel: +82-33-258-9262, Fax: +82-33-258-9097, E-mail: sora.baek@kangwon.ac.kr

ORCID: Jayoon Choi (https://orcid.org/0000-0001-6860-5223); Sora Baek (https://orcid.org/0000-0003-3404-6202); Gowun Kim (https://orcid. org/0000-0002-5694-8111); Hee-won Park (https://orcid.org/0000-0002-7434-6675).

(c) This is an open-access article distributed under the terms of the Creative Commons Attribution Non-Commercial License (http://creativecommons.org/ licenses/by-nc/4.0) which permits unrestricted noncommercial use, distribution, and reproduction in any medium, provided the original work is properly cited. Copyright $\odot 2021$ by Korean Academy of Rehabilitation Medicine 
be serious. It was ranked as the third leading cause of death in Korea in 2018 [3] and fourth worldwide in 2016 [4]. Swallowing difficulty is also a known risk factor of pneumonia. Both endotracheal aspiration and laryngeal penetration confirmed by videofluoroscopic swallowing study (VFSS) were significantly related to increased incidence of pneumonia [5].

Hence, protective methods need to be devised to reduce the risk of pneumonia, including pneumococcal vaccination [2]. Cough is the defensive mechanism of the body that removes foreign materials from the airway, and therefore, prevents aspiration. Reduced cough strength was reported in patients with pneumonia [6]. The voluntary peak cough flow (PCF) measured using a cough flow meter is a non-invasive and convenient way to measure the cough strength [7].

Reduced cough strength also showed association with swallowing difficulty. Voluntary cough flow readings were significantly low in patients with endotracheal aspiration or laryngeal penetration on VFSS or fiberoptic endoscopic examination of swallowing compared to the nondysphagic population (mean cough flow, 118.2 vs. 337.2 $\mathrm{L} / \mathrm{min}$ ) $[8,9]$. Among patients with dysphagia, reduced cough flow was associated with a higher risk of pneumonia $[6,10]$. However, in the coexistence of swallowing difficulty and reduced cough strength, their independent association with pneumonia needs to be investigated.

Therefore, the exact relationship between cough strength, dysphagia, and pulmonary complications needs to be explored through further research in order to understand their clinical applicability. We hypothesized that cough strength and presence of dysphagia are independently associated with the development of pneumonia. Here, we aimed to evaluate the possible risk factors for pneumonia and provide supporting data in guidelines that will be helpful in the prediction and prevention of pulmonary complications.

\section{MATERIALS AND METHODS}

\section{Participants}

This retrospective chart review included patients (both inpatients and outpatients) who underwent VFSS at the Kangwon National University Hospital between 23 July 2012 and 12 June 2019. Patients who did not perform the voluntary PCF test because of tracheostomy or poor cooperation were excluded. A total of 1,352 VFSS evaluations and PCF measurements of 821 patients were performed during the above-mentioned time period. Patients with pneumonia within 1 month from the date of VFSS were classified into the pneumonia group. The occurrence of pneumonia was defined through medical chart review as per the following criteria: (1) diagnosis of pneumonia or pneumonitis (International Classification of Diseases-10 [ICD-10] codes: J10.0, J11.0, J12.X, J13, J14, J15.X, J16.X, J17.0, J17.1, J17.8, J18.X, and J69.X) or (2) confirmed by both: blood or sputum culture and the chart review. The occurrence of pneumonia was subclassified as (1) pneumonia before VFSS test, "pneumoniaVFSS" and (2) pneumonia after VFSS, "VFSS-pneumonia"

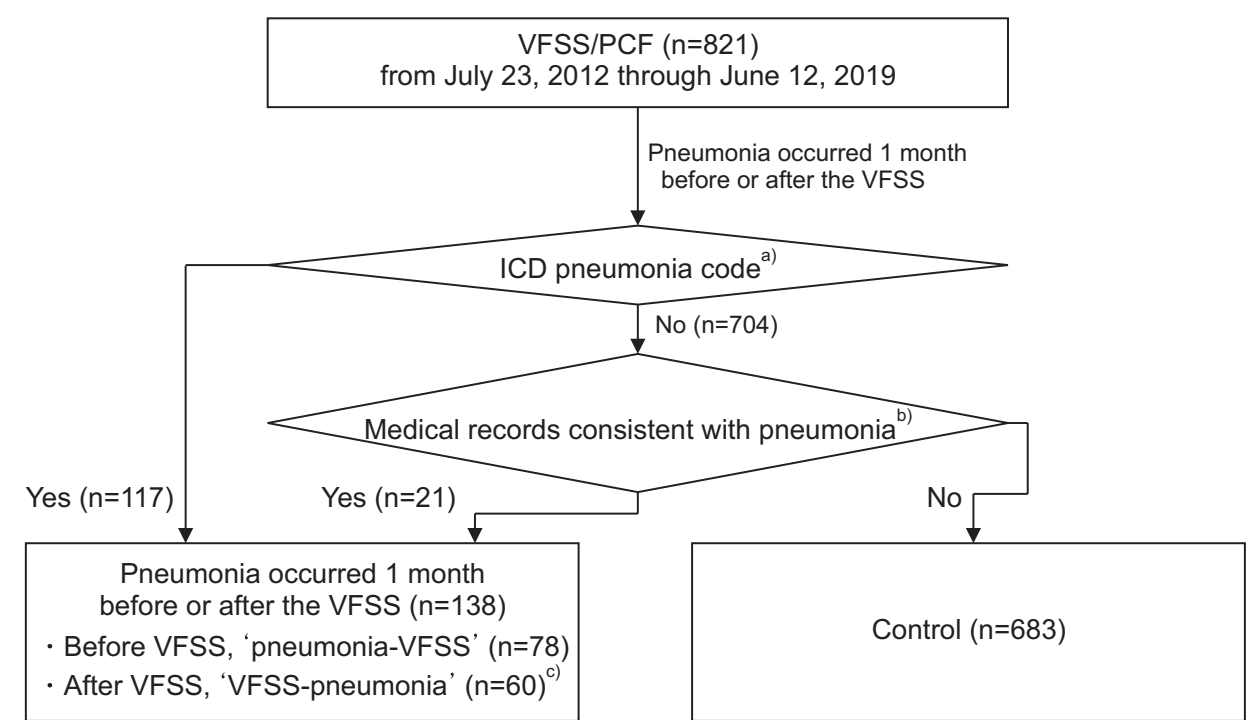

Fig. 1. Flow chart of participants enrollment in the study. VFSS, videofluoroscopic swallowing study; PCF, peak cough flow; ICD, International Classification of Diseases. ${ }^{\text {a) } J 10.0, ~ J 11.0, ~ J 12 . X, ~ J 13, ~}$ J14, J15.X, J16.X, J17.0, J17.1, J17.8, J18.X, and J69.X. ${ }^{\text {b) }}$ Confirmed by both blood or sputum culture and the chart review. ${ }^{c)}$ No significant differences in patient characteristics between pneumonia-VFSS and VFSS-pneumonia groups. 
(Fig. 1). If a patient with pneumonia had multiple VFSSs, the VFSS closest to the date of diagnosis of pneumonia was selected for the analysis. If a patient without pneumonia (control group) underwent multiple VFSSs, the earliest VFSS evaluation was included in the analysis.

Sex, age ( $<65$ and $\geq 65$ years), preceding conditions, and results of Korean version of modified Barthel Index (KMBI) [11] and Korean version of Mini-Mental State Examination (MMSE-K) [12] were collected from the medical records. The preceding conditions were categorized into three subgroups: (1) brain lesions, (2) neurodegenerative diseases, and (3) other miscellaneous diseases. Brain lesions included ischemic infarction, traumatic and non-traumatic hemorrhage, encephalopathy, brain tumor, hydrocephalus, arterio-venous malformation, status epilepticus, and cerebral palsy. Among the neurodegenerative diseases were parkinsonism and dementia. Other miscellaneous diseases included neuromuscular disease (NMD), cervical spine operation, other orthopedic surgery, oropharyngeal or mediastinal problems, facial or vocal fold palsy, pneumonia, medical problems other than pneumonia, and dysphagia of unknown origin.

The study protocol was approved by the Institutional Review Board of Kangwon National University Hospital (No. KNUH-A-2020-04-002). The informed consent was waived.

\section{Coughing ability}

Voluntary PCF measurements were collected through the retrospective chart review and were used to assess the coughing ability. The PCF test was conducted by physicians using a peak cough flow meter (Philips Personal Best Full Range Flow Meter; Respironics Inc., Parsippany, NJ, USA). The patient was seated in an upright position, instructed to breathe in deeply and cough as hard as possible. The PCF test was conducted three times, and the maximum value was selected for the analysis. We referred to the previous literature, and PCF was categorized as $\geq 270, \geq 160$ and $<270$, and $<160 \mathrm{~L} / \mathrm{min}$ [13].

\section{Swallowing function}

We collected the data of VFSS evaluation through the retrospective chart review. The presence of aspiration/ penetration during VFSS was used to assess the swallowing function. VFSS was performed by physicians and assisted by radiation technologists. The patients were seated in an upright position. The VFSS diet consisted of thin and thickened fluid, curd-type yogurt, pudding, rice gruel, and rice. All diets were impregnated with barium. A thickener (NUCARE Toromi Perpect; Daesang Life Science Corp., Seoul, Korea) was used following the manufacturer's guidelines. A test was discontinued if a large amount of aspiration was observed. All tests were recorded and evaluated with the 8-point PenetrationAspiration Scale (PAS) [14]. The largest score of PAS in any diet tested was selected for the analysis, and the PAS score was categorized as no dysphagia group (no presence of aspiration and penetration; PAS $=1$ ) and dysphagia group (presence of aspiration or penetration; PAS $\geq 2$ ). This scoring process was done by two physicians based on fluoroscopic images. If the scores were not agreed upon, a single score was achieved through the discussion of two physicians.

\section{Statistical analysis}

Baseline characteristics, coughing ability, and swallowing function between pneumonia-VFSS and VFSS-pneumonia subgroups were compared using the Pearson chisquare test, the likelihood ratio, and the Student t-test. If no statistical differences between the two subgroups were observed, they were combined into a single pneumonia group.

In order to make a comparison between the pneumonia and control groups, the Pearson chi-square test, the likelihood ratio, and the Student t-test were used. p-values were determined using the Pearson chi-square test (sex, age, presence of aspiration/penetration, and PCF), likelihood ratio test (preceding conditions) or the Student ttest (MBI and MMSE).

The Pearson chi-square test was used to investigate the association between dysphagia and PCF. Logistic regression analysis was used to investigate the associated factors of pneumonia. Variables with p-value $<0.05$ in the Pearson chi-square test, the likelihood ratio, and the Student t-test were included in univariable logistic regression analysis. Variables with $p$-value $<0.05$ in the univariable logistic regression analysis were included in the multivariable logistic regression analysis, and the backward input method was used. All analyses were performed using SPSS version 26.0 (IBM, Armonk, NY, USA), and statistical significance was defined as a p-value of $<0.05$. 


\section{RESULTS}

\section{Patient characteristics}

Out of 138 patients with pneumonia, 78 patients had pneumonia before VFSS (pneumonia-VFSS subgroup), and 60 patients had pneumonia after VFSS (VFSS-pneumonia subgroup). VFSS-pneumonia subgroup showed higher proportion of low PCF cases $(<160 \mathrm{~L} / \mathrm{min})(80 \%$ of 60 ) than the pneumonia-VFSS subgroup $(62.8 \%$ of 78 ), but the difference was not statistically significant $(\mathrm{p}=0.060)$. There were no other significant differences in characteristics between the two subgroups; therefore, both subgroups were combined as a single pneumonia group in further analysis.

The baseline characteristics of pneumonia $(n=138)$ and control $(n=638)$ groups are presented in Table 1. Of all patients $(\mathrm{n}=821), \mathrm{K}-\mathrm{MBI}$ and MMSE-K were reviewed in 470 and 351 patients, respectively. Certain characteristics were found to be different between the pneumonia and control groups. The pneumonia group was more likely to be male $(n=108 ; 78.3 \%)$, aged $>65$ years $(n=121 ; 87.7 \%)$, with neurodegenerative diseases as preceding condition ( $\mathrm{n}=25 ; 18.1 \%)$, and in poor functional level with lower value of K-MBI (39.1 \pm 26.59$)$; however, the score of MMSE-K was not significantly different. The pneumonia group also showed more dysphagia rate ( $\mathrm{n}=114 ; 82.6 \%)$, meaning that their swallowing function was poorer than that of the control group, and a higher proportion of patients had PCF value $<160 \mathrm{~L} / \mathrm{min}(\mathrm{n}=97 ; 70.3 \%)$.

\section{Dysphagia and peak cough flow}

The association between dysphagia and PCF was also analyzed in this study. Consistent with the findings of previous studies, patients with dysphagia $(n=554)$ were more likely to have a reduced value of PCF than patients without dysphagia $(n=267)$. The proportion of patients with PCF value $<160 \mathrm{~L} / \mathrm{min}$ was $62.8 \%$ in the patients with dysphagia, whereas it was only $39.0 \%$ in the patients without dysphagia. Detailed descriptions are present in Table 2.

\section{Risk factors related with pneumonia}

Sex, age, preceding conditions, score of K-MBI, presence of aspiration/penetration, and PCF subgroups were included in univariable logistic regression analysis. In univariable logistic regression analysis, male sex (odd ratio $[\mathrm{OR}]=2.31 ; 95 \% \mathrm{CI}, 1.50-3.56$ ), age $>65$ years $(\mathrm{OR}=2.30$; 95\% CI, 1.35-3.94), preceding conditions (neurodegenerative diseases, $\mathrm{OR}=3.86 ; 95 \% \mathrm{CI}, 2.23-6.68$; other miscellaneous diseases, $\mathrm{OR}=2.39 ; 95 \% \mathrm{CI}, 1.58-3.61$ ),

Table 1. Baseline characteristics between pneumonia and control groups

\begin{tabular}{|c|c|c|c|}
\hline & Control $(n=683)$ & Pneumonia $(\mathrm{n}=138)$ & p-value \\
\hline Sex, male & $416(60.9)$ & $108(78.3)$ & $<0.001^{\text {a) }}$ \\
\hline Age, $\geq 65 \mathrm{yr}$ & $516(75.5)$ & $121(87.7)$ & $0.002^{\mathrm{a})}$ \\
\hline Preceding conditions & & & $<0.001^{\text {b) }}$ \\
\hline Brain lesions & $476(69.7)$ & $63(45.7)$ & \\
\hline Neurodegenerative disease & $49(7.2)$ & $25(18.1)$ & \\
\hline Other disease & $158(23.1)$ & $50(36.2)$ & \\
\hline $\operatorname{MBI}(\mathrm{n}=470)$ & $48.2 \pm 30.38(n=419)$ & $39.1 \pm 26.59(n=51)$ & $0.042^{\mathrm{c})}$ \\
\hline $\operatorname{MMSE}(\mathrm{n}=351)$ & $20.4 \pm 6.47(n=316)$ & $19.3 \pm 8.55(\mathrm{n}=35)$ & $0.453^{\mathrm{c})}$ \\
\hline Presence of dysphagia & & & $<0.001^{\mathrm{a})}$ \\
\hline No dysphagia (no aspiration/penetration) & $243(35.6)$ & $24(17.4)$ & \\
\hline Dysphagia (aspiration or penetration) & $440(64.5)$ & $114(82.6)$ & \\
\hline PCF (L/min) & & & $<0.001^{\text {a) }}$ \\
\hline$\geq 270$ & $79(11.6)$ & $7(5.1)$ & \\
\hline$\geq 160$ and $<270$ & $249(36.5)$ & $34(24.6)$ & \\
\hline$<160$ & $355(52.0)$ & $97(70.3)$ & \\
\hline
\end{tabular}

Values are presented as number (\%) or mean \pm standard deviation.

MBI, modified Barthel Index; MMSE, Mini-Mental State Examination; PCF, peak cough flow.

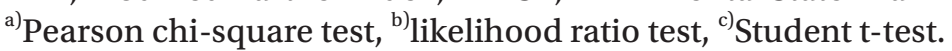


Table 2. Association between dysphagia and PCF

\begin{tabular}{lccc}
\hline & $\begin{array}{c}\text { No dysphagia } \\
(\mathbf{n}=267)\end{array}$ & $\begin{array}{c}\text { Dysphagia } \\
(\mathbf{n}=\mathbf{5 5 4})\end{array}$ & p-value \\
\hline $\mathrm{PCF}(\mathrm{L} / \mathrm{min})$ & & & $<0.001^{\mathrm{a})}$ \\
$\geq 270$ & $44(16.5)$ & $42(7.6)$ & \\
$\geq 160$ and $<270$ & $119(44.6)$ & $164(29.6)$ & \\
$<160$ & $104(39.0)$ & $348(62.8)$ & \\
\hline
\end{tabular}

Values are presented as number (\%).

PCF, peak cough flow.

${ }^{\text {a) }}$ Pearson chi-square test.

Table 3. Risk factors for pneumonia by univariable logistic regression analysis

\begin{tabular}{|c|c|c|}
\hline & OR (95\% CI) & p-value \\
\hline \multicolumn{3}{|l|}{ Sex } \\
\hline Female & 1 & \\
\hline Male & $2.31(1.50-3.56)$ & $<0.001$ \\
\hline \multicolumn{3}{|l|}{ Age group (yr) } \\
\hline$<65$ & 1 & \\
\hline$\geq 65$ & $2.3(1.35-3.94)$ & 0.002 \\
\hline \multicolumn{3}{|l|}{ Preceding conditions } \\
\hline Brain lesions & 1 & \\
\hline Neurodegenerative disease & $3.86(2.23-6.68)$ & $<0.001$ \\
\hline Other disease & $2.39(1.58-3.61)$ & $<0.001$ \\
\hline MBI & $0.99(0.98-1.00)$ & 0.044 \\
\hline \multicolumn{3}{|l|}{ Presence of dysphagia } \\
\hline $\begin{array}{l}\text { No dysphagia } \\
\text { (no aspiration/penetration) }\end{array}$ & 1 & \\
\hline $\begin{array}{l}\text { Dysphagia } \\
\text { (aspiration or penetration) }\end{array}$ & $2.62(1.64-4.19)$ & $<0.001$ \\
\hline \multicolumn{3}{|l|}{ PCF (L/min) } \\
\hline$\geq 270$ & 1 & \\
\hline$\geq 160$ and $<270$ & $1.54(0.66-3.61)$ & 0.320 \\
\hline$<160$ & $3.08(1.38-6.90)$ & 0.006 \\
\hline
\end{tabular}

MBI, modified Barthel index; PCF, peak cough flow; OR, odd ratio; $\mathrm{CI}$, confidence interval.

score of MBI (OR=0.99; 95\% CI, 0.98-1.00), presence of dysphagia (OR=2.62; 95\% CI, 1.64-4.19), and PCF <160 L/ $\min (\mathrm{OR}=3.08$; 95\% CI, 1.38-6.90) showed significant ORs (Table 3).

In multivariable logistic regression analysis, male sex (OR=6.62; 95\% CI, 2.70-16.26), other miscellaneous diseases (OR=2.52; 95\% CI, 1.14-5.58), presence of dysphagia (OR=3.82, 95\% CI, 1.42-10.23), and PCF $<160 \mathrm{~L} / \mathrm{min}$ (OR=14.34; 95\% CI, 1.84-111.60) were statistically signifi-
Table 4. Risk factors for pneumonia by multivariable logistic regression analysis (backward conditional input)

\begin{tabular}{lcc}
\hline & OR (95\% CI) & p-value \\
\hline $\begin{array}{l}\text { Sex } \\
\text { Female }\end{array}$ & 1 & \\
$\quad$ Male & $6.62(2.70-16.26)$ & $<0.001$ \\
Preceding conditions & & \\
$\quad$ Brain lesions & 1 & \\
$\quad$ Neurodegenerative disease & $1.86(0.70-4.96)$ & 0.212 \\
\hline $\begin{array}{l}\text { Other disease } \\
\text { Presence of dysphagia }\end{array}$ & $2.52(1.14-5.58)$ & 0.023 \\
$\begin{array}{l}\text { No dysphagia } \\
\text { (no aspiration/penetration) }\end{array}$ & & \\
$\begin{array}{l}\text { Dysphagia } \\
\text { (aspiration or penetration) }\end{array}$ & $3.82(1.42-10.23)$ & 0.008 \\
PCF (L/min) & & \\
$\geq 270$ & 1 & \\
$\geq 160$ and $<270$ & $4.82(0.60-38.56)$ & 0.138 \\
$<160$ & $14.34(1.84-111.6)$ & 0.011 \\
\hline
\end{tabular}

PCF, peak cough flow; OR, odd ratio; CI, confidence interval.

cant associated factors of pneumonia. The ORs and pvalues are shown in Table 4.

\section{DISCUSSION}

We hypothesized that the value of voluntary PCF and the presence of dysphagia were independently associated with the development of pneumonia. In this study, the significant independent association factors of pneumonia were male sex, preceding conditions other than brain lesions or neurodegenerative diseases, presence of dysphagia, and PCF $<160 \mathrm{~L} / \mathrm{min}$.

Coughing ability has been reported to be associated with an increased risk of pneumonia in patients with dysphagia [10]. In the research of Kulnik et al. [6], it was found that $1 \mathrm{~L} / \mathrm{min}$ increase in voluntary PCF reduced the risk of pneumonia by $0.6 \%$ through the logistic regression analysis. Hammond et al. [8] conducted a research in patients with ischemic stroke to predict the aspiration risk using voluntary cough, and this was associated with an aspiration risk with a cut-off value of $<2.9 \mathrm{~L} / \mathrm{s}$ ( $=174 \mathrm{~L} /$ min). Sakai et al. [15] also suggested in their study that PCF could be used as an indicator to evaluate the aspiration risk in older adults with pneumonia, and the cut-off 
value was $190 \mathrm{~L} / \mathrm{min}$.

Our study also revealed that dysphagia confirmed by the presence of aspiration/penetration in VFSS was significantly associated with the presence of pneumonia, with an OR of 3.82 in the multivariable analysis. This result was consistent with that of previous studies regarding the relationship between dysphagia and pneumonia. Dysphagia is known to be a significant risk factor for pneumonia in older adults in numerous studies [16-19]. A prospective study evaluated the risk factors for aspiration pneumonia in the older patients; $81 \%$ of the aspiration pneumonia group had oropharyngeal dysphagia, and this proportion was significantly higher than that in the control group [19]. Furthermore, a literature review has shown a relationship between oropharyngeal dysphagia and pneumonia and an association between the treatment of dysphagia with improved morbidity and mortality [20].

Dysphagia and reduced PCF showed a close relationship with pneumonia. Interestingly, swallowing function and respiratory function seem closely related as well. In our study, we found a statistically significant association between the presence of dysphagia and the reduced value of PCF. This result is consistent with that of previous studies. Min et al. [21] found that respiratory functions, which were assessed by PCF, forced vital capacity, and forced expiratory volume in a 1-second measure, had a significant correlation with dysphagia. Furthermore, there were many similar studies conducted, especially in patients with stroke. Stroke patients with dysphagia even had a lower value of voluntary cough ability than stroke patients without dysphagia [22]. In our study, we attempted to reveal the independent association of dysphagia and coughing ability with pneumonia. Through multivariable analysis, we showed that the value of voluntary PCF and the presence of dysphagia had independent associations with pneumonia. To the best of our knowledge, this is the first report about this relationship.

In our study, the patients with pneumonia tended to be males, and additionally, multivariable logistic regression analysis identified that male sex was a significant independent predictor of pneumonia. This result is consistent with that of previous studies. A study on individuals from nursing homes showed that male sex was associated with increased risk of pneumonia (OR was 1.98 in the univariable model and 1.9 in the multivariable model)
[2]. This result can be interpreted with the demographic characteristics of the male sex. Generally, the prevalence of smokers is higher in men than in women. In 2015, $40.3 \%$ of the global male population aged $\geq 15$ years were tobacco users [23]. Smoking increases the risk of pneumonia and death from pneumonia $[24,25]$. Similar to smoking, the prevalence of alcohol consumption is also higher in the male population than in the female population. In 2016, the prevalence of current drinking in the male population was $53.6 \%$, whereas it was $32.3 \%$ in the female population [26]. Koivula et al. [27] revealed that alcoholism was an independent risk factor for pneumonia by multivariable logistic regression analysis (relative risk=9.0; 95\% CI, 5.1-16.2). Presumably, the higher proportion of tobacco use and alcohol consumption in the male population could play a major role in increasing the risk of pneumonia than in females.

In our study, the data regarding the preceding conditions of patients were collected and analyzed to investigate the possible association with the risk of pneumonia. The preceding conditions were categorized into three subgroups. The most common subgroup was the brain lesions subgroup, which included those with ischemic cerebral infarction. Pneumonia is a well-known and significant medical complication after stroke, and the association between cough function impairment and stroke has also been widely reported $[8,28]$. However, contrary to our expectation, the patients with pneumonia tended to have neurodegenerative diseases or other miscellaneous diseases as a preceding condition.

In other miscellaneous disease subgroups, there were NMDs, cervical spine operation, other orthopedic surgeries, oropharyngeal or mediastinal problems, facial or vocal fold palsy, pneumonia, medical problems other than pneumonia, and dysphagia of unknown origin. NMDs, such as Duchenne muscular dystrophy, amyotrophic lateral sclerosis, and other NMDs, have been reported for their association with pneumonia $[13,29]$. Most of the NMDs in our study either included amyotrophic lateral sclerosis, dermatomyositis, or myopathy. Cervical spine operation [30] and oropharyngeal/esophageal or mediastinal problems induced by tumorous lesions, such as tongue cancer [31] or lymphoma [32], could provoke structural or functional change. Facial [33] or vocal fold palsy [34] could also affect swallowing function. All of the above conditions could lead to the incidence of dyspha- 
gia and aspiration, and therefore, could be associated with the incidence of pneumonia as well. Chronic lung diseases have also mentioned as a risk factor of pneumonia $[27,35]$.

Since impaired coughing ability was found to be an independent association factor for the development of pneumonia, measuring cough flow in patients at high risk for pneumonia may be a useful assessment. PCF meter could be the instrument of choice for measurement. It is simple, non-invasive, inexpensive, and easy to handle. Patients in this study were categorized into three subgroups on the basis of their coughing ability, and these subgroups were defined according to the previous literature [13]. However, in further studies, more detailed categorization would be required to select the patients at high risk of pneumonia.

There were some limitations in this study. First, this study was a retrospective study at a single institution. The nature of the study may have lead to a potential bias. It could also be presumed that the swallowing function and cough strength would have a strong association with aspiration pneumonia. However, since aspiration pneumonia is presumptively diagnosed based on the aspiration history and its antecedent relationship with pneumonia, it was difficult to confirm the aspiration event in this retrospective study. Therefore, we decided to expand the targeted subject to overall pneumonia. Second, the functional status of the patients included in the analysis was relatively not severe. Only patients who were capable of following the instructions and participating in the voluntary PCF test and VFSS were included, but patients who had poor functional ability and could not perform the test were excluded. Third, preceding conditions were found to be the significant association factor of pneumonia. This study consisted of a heterogeneous group of patients who were classified into three subgroups, including brain lesions, neurodegenerative diseases, and other miscellaneous diseases. These subgroups should be further subdivided into smaller subgroups or the disease entity itself to identify a more accurate relationship between pneumonia and the preceding conditions. Especially, since the other miscellaneous disease group was found to be a major association factor of pneumonia, the detailed component of this group should have been explored and discussed in the study. However, this group was too heterogeneous, and the sample size was too small such that the analysis of the group was very difficult. Therefore, this group was analyzed as it was in this study, but it should be noted that a more detailed segmentation of this group is required in the future study. Finally, social history, such as smoking or drinking and environmental or occupational history, were not collected in this study. Since we predicted that the association between male sex and pneumonia would be induced by smoking and drinking, more information should be collected for a precise analysis. Therefore, these issues should be kept in consideration for further researches.

In conclusion, this study identified that the presence of dysphagia and impaired coughing ability with PCF $<160$ $\mathrm{L} /$ min showed a significant relationship with the development of pneumonia as well as male sex and preceding conditions. Impairment in swallowing and coughing function may be the independent risk factors for the development of pulmonary complications. We recommend that physicians measure cough flow in patients at the risk of pneumonia regardless of the presence of dysphagia. Patients with PCF $<160 \mathrm{~L} / \mathrm{min}$ require more attention with lung care and should be encouraged with the usage of voluntary coughing strategy to prevent possible pulmonary complications.

\section{CONFLICT OF INTEREST}

No potential conflict of interest relevant to this article was reported.

\section{AUTHOR CONTRIBUTION}

Conceptualization: Baek S, Choi J, Kim G, Park HW. Methodology: Baek S, Choi J. Writing - original draft: Baek S, Choi J. Writing - review and editing: Baek S, Choi J. Approval of final manuscript: both authors.

\section{REFERENCES}

1. Choi MJ, Song JY, Noh JY, Yoon JG, Lee SN, Heo JY, et al. Disease burden of hospitalized communityacquired pneumonia in South Korea: analysis based on age and underlying medical conditions. Medicine (Baltimore) 2017;96:e8429.

2. Loeb M, McGeer A, McArthur M, Walter S, Simor AE. Risk factors for pneumonia and other lower respira- 
tory tract infections in elderly residents of long-term care facilities. Arch Intern Med 1999;159:2058-64.

3. Statistics Korea. The Annual Report on the Causes of Death Statistics 2019. Daejeon, Statistics Korea; 2020.

4. World Health Organization. The top 10 causes of death [Internet]. Geneva, World Health Organization; 2020 [cited 2021 Nov 1]. Available from: https://www. who.int/news-room/fact-sheets/detail/the-top-10causes-of-death.

5. Pikus L, Levine MS, Yang YX, Rubesin SE, Katzka DA, Laufer I, et al. Videofluoroscopic studies of swallowing dysfunction and the relative risk of pneumonia. AJR Am J Roentgenol 2003;180:1613-6.

6. Kulnik ST, Birring SS, Hodsoll J, Moxham J, Rafferty GF, Kalra L. Higher cough flow is associated with lower risk of pneumonia in acute stroke. Thorax 2016;71:474-5.

7. Tzani P, Chiesa S, Aiello M, Scarascia A, Catellani C, Elia D, et al. The value of cough peak flow in the assessment of cough efficacy in neuromuscular patients: a cross sectional study. Eur J Phys Rehabil Med 2014;50:427-32.

8. Hammond CA, Goldstein LB, Horner RD, Ying J, Gray L, Gonzalez-Rothi L, et al. Predicting aspiration in patients with ischemic stroke: comparison of clinical signs and aerodynamic measures of voluntary cough. Chest 2009;135:769-77.

9. Plowman EK, Watts SA, Robison R, Tabor L, Dion C, Gaziano J, et al. Voluntary cough airflow differentiates safe versus unsafe swallowing in amyotrophic lateral sclerosis. Dysphagia 2016;31:383-90.

10. Bianchi C, Baiardi P, Khirani S, Cantarella G. Cough peak flow as a predictor of pulmonary morbidity in patients with dysphagia. Am J Phys Med Rehabil 2012;91:783-8.

11. Jung HY, Park BK, Shin HS, Kang YK, Pyun SB, Paik NJ, et al. Development of the Korean version of Modified Barthel Index (K-MBI): multi-center study for subjects with stroke. J Korean Acad Rehabil Med 2007;31:28397.

12. Park JH, Kwon YC. Modification of the mini-mental state examination for use in the elderly in a non-western society. Part 1: development of Korean version of mini-mental state examination. Int J Geriatr Psychiatry 1990;5:381-7.

13. Sahni AS, Wolfe L. Respiratory care in neuromuscular diseases. Respir Care 2018;63:601-8.

14. Rosenbek JC, Robbins JA, Roecker EB, Coyle JL, Wood JL. A penetration-aspiration scale. Dysphagia 1996;11:93-8.

15. Sakai Y, Ohira M, Yokokawa Y. Cough strength is an indicator of aspiration risk when restarting food intake in elderly subjects with community-acquired pneumonia. Respir Care 2020;65:169-76.

16. Manabe T, Teramoto S, Tamiya N, Okochi J, Hizawa N. Risk factors for aspiration pneumonia in older adults. PLoS One 2015;10:e0140060.

17. Marik PE, Kaplan D. Aspiration pneumonia and dysphagia in the elderly. Chest 2003;124:328-36.

18. van der Maarel-Wierink CD, Vanobbergen JN, Bronkhorst EM, Schols JM, de Baat C. Risk factors for aspiration pneumonia in frail older people: a systematic literature review. J Am Med Dir Assoc 2011;12:344-54.

19. Langmore SE, Terpenning MS, Schork A, Chen Y, Murray JT, Lopatin D, et al. Predictors of aspiration pneumonia: how important is dysphagia? Dysphagia 1998;13:69-81.

20. Hammond CA, Goldstein LB. Cough and aspiration of food and liquids due to oral-pharyngeal dysphagia: ACCP evidence-based clinical practice guidelines. Chest 2006;129(1 Suppl):154S-168S.

21. Min SW, Oh SH, Kim GC, Sim YJ, Kim DK, Jeong HJ. Clinical importance of peak cough flow in dysphagia evaluation of patients diagnosed with ischemic stroke. Ann Rehabil Med 2018;42:798-803.

22. Kimura Y, Takahashi M, Wada F, Hachisuka K. Differences in the peak cough flow among stroke patients with and without dysphagia. J UOEH 2013;35:9-16.

23. World Health Organization. WHO global report on trends in prevalence of tobacco use 2000-2025. 3rd ed. Geneva, Switzerland: World Health Organization; 2019.

24. Almirall J, Gonzalez CA, Balanzo X, Bolibar I. Proportion of community-acquired pneumonia cases attributable to tobacco smoking. Chest 1999;116:375-9.

25. Bello S, Menendez R, Antoni T, Reyes S, Zalacain R, Capelastegui A, et al. Tobacco smoking increases the risk for death from pneumococcal pneumonia. Chest 2014;146:1029-37.

26. World Health Organization. Global status report on alcohol and health 2018. Geneva, Switzerland: World Health Organization; 2018. 
27. Koivula I, Sten M, Makela PH. Risk factors for pneumonia in the elderly. Am J Med 1994;96:313-20.

28. Lee SJ, Lee KW, Kim SB, Lee JH, Park MK. Voluntary cough and swallowing function characteristics of acute stroke patients based on lesion type. Arch Phys Med Rehabil 2015;96:1866-72.

29. Burkhardt C, Neuwirth C, Sommacal A, Andersen $\mathrm{PM}$, Weber M. Is survival improved by the use of NIV and PEG in amyotrophic lateral sclerosis (ALS)? A post-mortem study of 80 ALS patients. PLoS One 2017;12:e0177555.

30. Anderson KK, Arnold PM. Oropharyngeal dysphagia after anterior cervical spine surgery: a review. Global Spine J 2013;3:273-86.

31. Son YR, Choi KH, Kim TG. Dysphagia in tongue can- cer patients. Ann Rehabil Med 2015;39:210-7.

32. Astudillo L, Peron JM, Sailler L, Danjoux M, RigalHuguet F, Arlet-Suau E. Dysphagia revealing esophageal involvement by non-Hodgkin's lymphoma. Ann Hematol 2005;84:482-3.

33. Moverare T, Lohmander A, Hultcrantz M, Sjogreen L. Peripheral facial palsy: speech, communication and oral motor function. Eur Ann Otorhinolaryngol Head Neck Dis 2017;134:27-31.

34. Ha JF. Unilateral vocal fold palsy \& dysphagia: a review. Auris Nasus Larynx 2020;47:315-34.

35. Almirall J, Rofes L, Serra-Prat M, Icart R, Palomera $\mathrm{E}$, Arreola V, et al. Oropharyngeal dysphagia is a risk factor for community-acquired pneumonia in the elderly. Eur Respir J 2013;41:923-8. 